\title{
Effect of a Novel Polyherbal Formulation on Diabetes Induced Memory Deficits in Rats
}

Prabhat Upadhyay ${ }^{1}$, Ananya Sadhu ${ }^{2 *}$, Suresh Purohit ${ }^{1}$, Praveen K Singh ${ }^{3}$, Shivapriya Shivakumar ${ }^{4}$, Aruna Agrawal ${ }^{5}$, K. Ilango ${ }^{4}$ and Govind Prasad Dubey ${ }^{2}$

${ }^{1}$ Department of Pharmacology, IMS, BHU, Varanasi, India

${ }^{2}$ Collaborative Programme, Institute of Medical Science, BHU, Varanasi, India

${ }^{3}$ Arvind Remedies Ltd. 190, Poonamallee high road, Aminjikarai, Chennai, Tamil Nadu-600084, India

${ }^{4}$ Interdisciplinary School of Indian System of Medicine (ISISM), SRM University, Kattankulathur -603203

${ }^{5}$ Department of Kriya Sharir, Faculty of Ayurveda, IMS, BHU, Varanasi, India

\begin{abstract}
Epidemiological, clinical and experimental studies have shown a strong correlation between Diabetes mellitus (DM) and neurodegenerative disorders. Type II diabetes mellitus (T2DM) leads to elevated levels of plasma glucose and causes neurological complications including impairment of learning and memory processes. The pathogenesis mainly includes metabolic dysfunction due to brain insulin resistance, oxidative stress, inflammation and vascular complications. In this study we explored a new pharmacological intervention by investigating the neuroprotective effects of a standardized polyherbal formulation (PF) [(Bacopa monnieri $(20 \mathrm{mg} / \mathrm{kg})$, Hippophae rhamnoides $(25$ $\mathrm{mg} / \mathrm{kg}$ ), and Dioscorea bulbifera (15 mg/kg), p.o] on passive avoidance learning (PAL) and memory in normal and streptozotocin (STZ) induced diabetic rats. Treatment was started after the onset of hyperglycemia following STZ (60 $\mathrm{mg} / \mathrm{kg}$, i.p) injection. PAL and memory were assessed 30 days after treatment, followed by retention test $24 \mathrm{~h}$ after training. At the beginning and end, body weights and blood glucose measurements of the animals were done. The results showed STZ induced diabetic rats had severe impairments in acquisition and retrieval processes of PAL and memory. Treatment with the PF significantly improved cognitive deficits, increased body weight and lowered plasma glucose levels in the treated as compared to untreated diabetic rats. We hypothesize that acetyl choline enhancing, antioxidant, hypoglycemic and hypolipidemic properties of phytomolecule such as bacosides, quercetin, and diosgenin in the PF might be responsible for the nootropic effects of polyherbal formulation. Our study highlights the therapeutic potential of this polyherbal formulation for multi-targeted treatment of diabetes induced cognitive impairment.
\end{abstract}

Keywords: Polyherbal formulation; Diabetes mellitus; Streptozotocin; Passive avoidance; Memory; Blood glucose; Cognitive impairment

\section{Introduction}

Growing evidence (experimental, clinical and epidemiological studies) have established that diabetes mellitus a chronic metabolic disorder is associated with neurological complications, cognitive impairments and lead to sporadic Alzheimer's disease (AD) or Type III diabetes [1-6]. It is posited that insulin / Insulin like Growth Factor (IGF) resistance in the brain results in starvation and disrupts downstream signalling pathways that results in neuronal death [7-9]. It initiates a cascade of neurodegenerative changes that is propagated by increased oxidative and endoplasmic reticulum (ER) stress, mitochondrial dysfunction, neuroinflammation, impaired energy metabolism, disrupted metabolic functions, and dysregulated lipid metabolism [10]. This cellular dysfunction result in AD specific molecular changes which include plaque formation, kinase activation that aberrantly phosphorylate tau protein and lead to accumulation of neurofibrillary tangles, accumulation of neurotoxic amyloid beta precursor protein peptides (AbPP-Ab) and cholinergic dyshomeostasis [11,12]. The prevalence rate of sporadic $\mathrm{AD}$ is alarming and is considered the most common cause of dementia in elderly [13]. Over the last decade the Food and Drug Administration (FDA) has not approved any new drugs for the treatment of $\mathrm{AD}$; those which are in the market include acetylcholineesterase (AChE) inhibitors - Donepezil, Rivastigmine, and Galantamine or glutamate receptor antagonist Memantine [14,15]. However these drugs have many side effects, provide only symptomatic relief and do not halt or delay the progression of the disease $[16,17]$. Hence, there is an urgent need to explore novel therapies.

Herbal drugs are gaining worldwide popularity because of lesser side effects, cost effectiveness and easy availability to poor people particularly in developing countries [18,19]. Ayurveda, the Indian system of medicine have utilised the medicinal properties of several herbs to improve memory and cognitive function and to treat neurodegenerative diseases [20-24]. Many studies have therefore, assessed the therapeutic potential of novel phytomolecules to improve cognition, special learning and memory in streptozotocin diabetic rat model that induces AD-type neurodegeneration [25-30]. Since AD is associated with multiple pathologies such as oxidative stress, neuroinflammation and deficiency of acetylcholine, hence it is advantageous to use a multi-targeted therapeutic approach for treatment [31-34]. Hence, taking lead from ancient wisdom our group has standardized a polyherbal formulation (PF) using hydro-alcoholic extracts of Bacopa monnieri, Hippophae rhamnoides and Dioscorea bulbifera and have recently reported its positive therapeutic effects in elderly AD patients with cognitive deficits in a clinical study [35].

In the present study we aimed to assess the nootropic effects of PF [36] on passive avoidance learning paradigm and study its effect on blood glucose levels and body weight in streptozotocin diabetic rat model. Streptozotocin (STZ) is a nitrosamine-related compound

*Corresponding author: Ananya Sadhu, Collaborative Programme, Institute of Medical Science, BHU, Varanasi, India, Tel: 0542-2369937; E-mail:Ananya.s.84@gmail.com

Received: September 12, 2015; Accepted: October 12, 2015; Published: October 14, 2015

Citation: Upadhyay P, Sadhu A, Purohit S, Singh PK, Shivakumar S, et al. (2015) Effect of a Novel Polyherbal Formulation on Diabetes Induced Memory Deficits in Rats. Clin Exp Pharmacol 5: 194. doi:10.4172/2161-1459.1000194

Copyright: (c) 2015 Upadhyay P, et al. This is an open-access article distributed under the terms of the Creative Commons Attribution License, which permits unrestricted use, distribution, and reproduction in any medium, provided the original author and source are credited. 
that induces brain insulin resistance and insulin deficiency which results in cognitive impairment and $\mathrm{AD}$-type neurodegeneration [37]. Therefore this animal model has been used to study potential therapeutic molecules for treatment of cognitive deficits induced by diabetes [6,38]. This is a novel study since earlier reports were mainly focused on monotherapy, while in this study we assessed the medicinal properties (e.g., anti-oxidant, anti-inflammatory, antihyperlipidemic, antihyperglycemic and neuromodulatory) of three plants in a combined herbal formulation [39-41]. We envisage that this study will be useful in exploring new multi- targeted treatment strategies to improve the neurological complications induced by diabetes.

\section{Methods}

\section{Drugs}

Streptozotocine (STZ) was purchased from (Sigma-Aldrich chemical Co. India) and dissolved in $1 \mathrm{ml}$ saline, immediately before use. Other chemicals, reagents and solvents were procured from Biotech India Private Ltd. and were of analytical grade.

\section{Animals}

Male Wistar rats with body weight of 200-250 g were procured from the central animal house of Banaras Hindu University. The animals were kept at $23^{\circ} \mathrm{C} \pm 2^{\circ} \mathrm{C}$ with a relative humidity at $50 \pm 10 \%$, in separate cages under 12-h light: 12 -h dark light cycle. They were given standard food pellets and water ad libitum, during the entire duration of the experiment. Each experimental group consisted of six animals. Animal ethical committee approval was taken and all procedures for treatments of laboratory animals were done in accordance with the criteria outlined in the Guide for the Care and Use of Laboratory Animals (National Institutes of Health (NIH) publication 86-23; revised 1985; http://www.oacu.od.nih.gov/regs/guide/guidex.htm). Prior to the experiment all animals were acclimatized to laboratory conditions.

\section{Experimental design}

The animals in this study were randomly divided into four groups $(n=6)$; two control [control $(C)$ and control group receiving polyherbal formulation $(\mathrm{C}+\mathrm{P})$ ] and two diabetic [diabetes $(\mathrm{D})$, diabetic group receiving polyherbal formulation $(\mathrm{D}+\mathrm{P})]$. The control rats received intra peritoneal injections of physiological saline. Diabetes was induced by a single i.p injection of STZ $(60 \mathrm{mg} / \mathrm{kg})$ prepared in citrate buffer, $\mathrm{pH} 4.5$, this experimental model has been validated by several previous studies [42-44]. Three days after STZ injection, fasting blood glucose was determined using blood glucose monitor (Accuchek; Roche, Mannheim, Germany). Animals were considered diabetic if plasma glucose levels exceeded $250 \mathrm{mg} / \mathrm{dl}$. After confirmation of diabetes the animals were orally administered polyherbal formulation [(Bacopa monnieri $(20 \mathrm{mg} / \mathrm{kg})$, Hippophae rhamnoides $(25 \mathrm{mg} / \mathrm{kg})$, and Dioscorea bulbifera $(15 \mathrm{mg} / \mathrm{kg})$ ] [36] using gavage needle to control and diabetic groups for 30 days. After the treatment period, learning and memory were evaluated using Passive avoidance learning (PAL) test. All animals were weighed and plasma glucose levels were determined at the beginning and end of the experiment.

\section{Behavioural test}

Passive avoidance learning (PAL) test: The step through passive avoidance test is based on contextual fear conditional learning [45] and is used to evaluate emotional memory. The animals learn to avoid a specific place associated with an aversive event. A reduction in stepthrough latency (STL) was used as an indication of impaired memory.
The apparatus consisted of one bright chamber $(20 \times 20 \times 30 \mathrm{~cm})$ made up of transparent plastic and one dark chamber $(20 \times 20 \times 30 \mathrm{~cm})$ with walls made of dark opaque plastic. These chambers were separated by a guillotine door $(6 \times 8 \mathrm{~cm})$. The floors of both chambers contained stainless steel rods spaced $0.5 \mathrm{~cm}$ apart and an electric shock generator was used to electrify the floor of the dark chamber and the test was performed as previously described [27,30].

Training: Animals in all the experimental groups were given two test trials to acclimatize them with the apparatus. The rat was first placed in the bright compartment facing away from the door, $5 \mathrm{~s}$ later the guillotine door was raised to allow the rat to explore and move into the dark compartment as a natural preference. Upon entering the dark compartment the door was closed and the rat was allowed to stay there for $30 \mathrm{~s}$ and then put back into its home cage. The habituation trial was repeated $30 \mathrm{~min}$ later and after the same interval the first acquisition trial was performed. The time taken by the rat to spontaneously enter the dark compartment was denoted as entrance latency (STL-a) and time was noted only when the animal had placed all four paws in the dark compartment. After entry, the guillotine door was closed and a mild electrical shock $(0.5 \mathrm{~mA})$ was applied for $3 \mathrm{~s}$. After $30 \mathrm{~s}$ the rat was returned to the home cage. This procedure was again repeated after $2 \mathrm{~min}$. The rat received a foot shock each time it entered the dark compartment. Training was terminated when the rat remained in the bright compartment for $120 \mathrm{sec}$ and the number of trials, i.e., entry into the dark compartment was recorded.

Retention test: Twenty four hours later, the rat was placed in the bright compartment as in the PAL acquisition trial. The guillotine door was raised after $5 \mathrm{~s}$, the retention latency (STL-r) and time spent in the dark compartment (TDC) was recorded for up to $300 \mathrm{~s}$. The time taken for a mouse to enter the dark compartment after door opening was defined as latency. If the rat did not enter the dark chamber within 300 s, the retention test was terminated and a max score of $300 \mathrm{~s}$ was recorded.

\section{Statistical analysis}

One-Way ANOVA followed by Tukey as post hoc test was done to determine statistically significant differences between experimental groups. All the results were expressed as Mean \pm SEM (standard error of the mean) and statistical significance was considered at $\mathrm{p}<0.05$. All analyses of the data were performed by using Graph Prism Pad software version 5.0.

\section{Results}

\section{Effects of treatments on the PAL acquisition}

During first acquisition trial (before receiving electrical shock), the entrance latency time (STL-a) between the control and the diabetic groups were statistically insignificant $[(\mathrm{p}=0.8188)$, Figure $1 \mathrm{~A}]$. This indicated that rats in different experimental groups had similar exploratory behaviour in dark. However, we observed significant differences in the number of trials to acquisition criteria between control and diabetic experimental groups $[(p<0.0001)$, Figure 1B]. Tukey's Multiple Comparison test showed that number of trial to acquisition in control groups were significantly less than the diabetic groups. Number of trial in group $C$ was significantly lower compared to group $\mathrm{D}(\mathrm{P}<0.001)$ and $\mathrm{D}+\mathrm{P}(\mathrm{P}<0.05)$. Administration of polyherbal formulation resulted in no significant changes between groups $C$ vs. $\mathrm{C}+\mathrm{P}(\mathrm{p}>0.05)$; however, there was significant decrease in the trial number between groups $\mathrm{D}$ vs. $\mathrm{D}+\mathrm{P}(\mathrm{P}<0.05)$. 

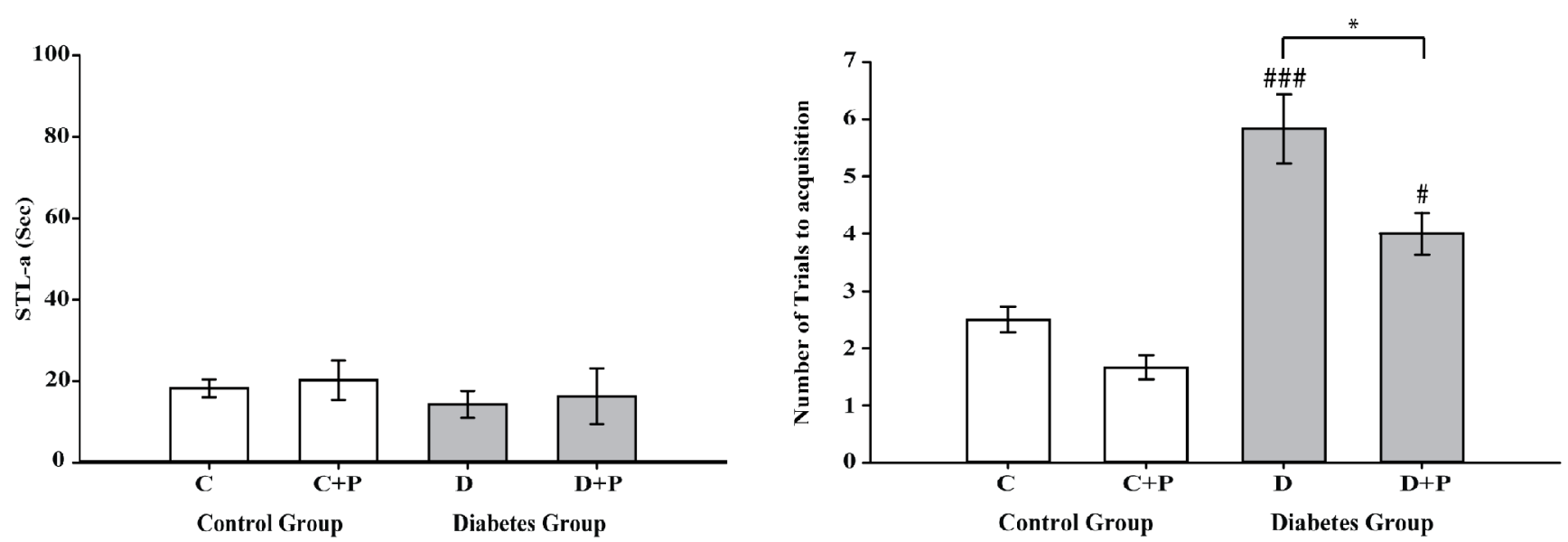

Figure 1: Effect of oral administration of polyherbal formulation on PAL test in acquisition trial.

Passive avoidance learning (PAL) test was performed following 30 day treatment period with saline $(C)$ and saline + polyherbal formulation $(C+P)$ to control groups and with streptozotocin and streptozotocin + polyherbal formulation (D+P) for diabetic groups.

A. Graph represents step-through latency in the acquisition trial (STL-a) of passive avoidance learning (PAL) task in the control and diabetic groups. Columns represent Mean \pm SEM of step-through latency time (s).

B. Graph represents number of trials to acquisition of passive avoidance learning (PAL) task in the control and diabetic groups. Columns represent mean \pm SEM.

Tukey's multiple comparison tests was done to compare values between groups. ${ }^{* *}$ indicates $\mathrm{P}<0.001,{ }^{* *}$ indicates $\mathrm{P}<0.01,{ }^{*}$ indicates $\mathrm{P}<0.05$ and $\mathrm{ns}$ indicates

$P>0.05$. \# indicates comparison of all groups with control group $C$

\section{Effects of treatments on the PAL retention}

The PAL retention test was conducted $24 \mathrm{~h}$ after training process. We observed significant difference in the retention latency time (STL-r) between the experimental groups $(\mathrm{P}<0.0001)$. The STL-r time for group $\mathrm{C}$ was significantly greater than group $\mathrm{D}(\mathrm{P}<0.001)$ and $\mathrm{D}+\mathrm{P}$ $(\mathrm{P}<0.01)$; however administration of $\mathrm{PF}$ significantly increased the retention latency in group $\mathrm{C}+\mathrm{P}$ vs. group $\mathrm{C}(\mathrm{P}<0.05)$. In addition, we observed a major difference in STL-r between saline treated diabetic group $\mathrm{D}$ compared to group $\mathrm{D}+\mathrm{P}$ that received $\mathrm{PF}(\mathrm{P}<0.01)$. We also observed statistically significant differences in time spend in the dark compartment (TDC) between the control and diabetic groups $[(\mathrm{P}<0.0001)$ (Figure $2 \mathrm{~B})]$. Tukey's multiple comparison test showed that TDC in saline treated control group $C$ was significantly less compared to the saline treated diabetic group $\mathrm{D}(\mathrm{P}<0.001)$ and $\mathrm{PF}$ treated diabetic group $\mathrm{D}+\mathrm{P}(\mathrm{P}<0.05)$. This result was consistent with finding that diabetes induces cognitive impairment. However, upon $\mathrm{PF}$ treatment TDC in diabetic group $\mathrm{D}+\mathrm{P}$ was found to be significantly lower than saline treated diabetic group $\mathrm{D}(\mathrm{P}>0.05)$ due to improvement in memory and retention of learned behaviour.

\section{Effects of treatments on the body weight and plasma glucose}

We analysed the blood glucose levels of rats in different experimental groups at the beginning and end of the treatment period (Figure 3A). Statistical analysis by one way ANOVA showed that there was no significant differences between the control and diabetic group at the beginning $(\mathrm{P}=0.828)$. We performed similar analysis for the experimental groups at the end of 30 days treatment and observed statistically significant differences in plasma glucose levels between the control and diabetic groups $(\mathrm{P}<0.0001)$. Tukey post hoc test revealed that plasma glucose levels of diabetic group $\mathrm{D}$ was significantly higher than control group $\mathrm{C}$ and $\mathrm{PF}$ treated group $\mathrm{C}+\mathrm{P}(\mathrm{P}<0.001)$. Additionally, analysis showed that administration of $\mathrm{PF}$ to diabetic rats significantly reduced plasma glucose levels (group $\mathrm{D}+\mathrm{P}$ ) in comparison with untreated diabetic rats in group $\mathrm{D}(\mathrm{P}<0.001)$. We also compared the body weight of the rats between groups at the beginning and end of 30 days treatment period (Figure 3B). We observed no significant changes in the body weight between the control and diabetic groups at the beginning of the study $(\mathrm{P}=0.201)$ by one way ANOVA analysis. Similar analysis was done for comparing body weight among experimental groups at the end of the experiment and we observed a significant reduction in the body weight of rats in the saline treated diabetic group $\mathrm{D}$ compared to the saline treated control group $\mathrm{C}(\mathrm{P}<0.001)$. However, $\mathrm{PF}$ treated diabetic rats showed significant increase in body weight as compared to untreated diabetic rats $(\mathrm{P}<0.01)$.

\section{Discussion}

Several experimental and clinical studies have established a strong association of diabetes with neurodegenerative disorders such $\mathrm{AD}$ [46]. It is proven that the molecular and biochemical features of sporadic $\mathrm{AD}$ correspond with features of Type 1 (insulin deficiency) and Type 2 (insulin resistance) diabetes; therefore $\mathrm{AD}$ is essentially a metabolic disease which is also referred to as Type 3 diabetes [3]. It is established that insulin and Insulin like Growth Factor (IGF) resistance in the brain affects glucose uptake and utilization that causes imbalance in energy production, leads to mitochondrial dysfunction, oxidative and ER stress that propagates cell death cascades, neuroinflammation, dysregulation of lipid metabolism leading to accumulation of toxic lipids (ceramides) and cholinergic dyshomeostasis [47-49]. At the molecular level, down regulation of insulin receptors (IR) at the blood brain barrier causes lower insulin transport to the brain, thus causing insulin resistance. Inappropriate activation of kinases result in inhibitory phosphorylation that affects PI3K and the MAPK insulin mediated signalling cascades, both of which are involved in the maintenance of synaptic plasticity and cell stress response $[2,7,50]$. These processes intensely effects neuronal survival by disrupting neurotransmitter homeostasis and neuronal cytoskeleton, causes accumulation of toxic oligomeric fibrils (neurofibrillary tangles) and insoluble aggregates (amyloid beta plaques) and compromise learning and memory functions [12]. These studies indicate the multifactorial pathogenesis of neurodegeneration that is caused due to insulin resistance and signals the need to assess innovative approaches for drug discovery and development.

In the present study streptozotocin was used to induce diabetes 

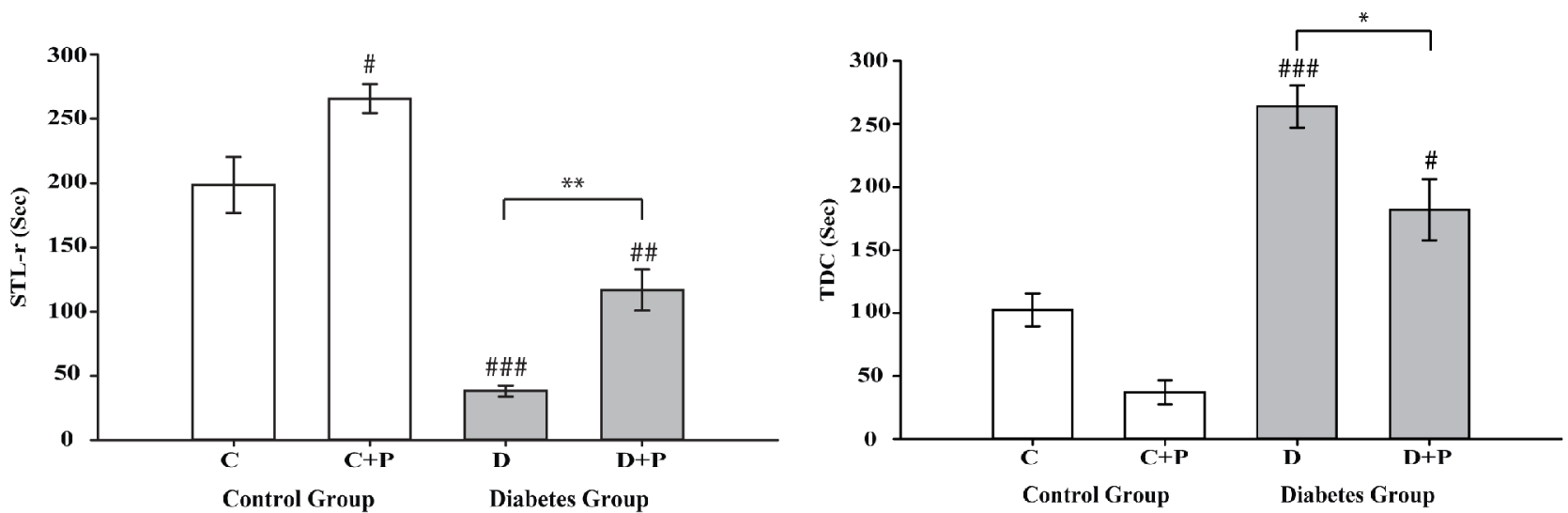

Figure 2: Effect of oral administration of polyherbal formulation on PAL test in retention trial.

Passive avoidance learning (PAL) test was performed following 30 days treatment period with saline $(\mathrm{C})$ and saline + polyherbal formulation $(\mathrm{C}+\mathrm{P})$ to control groups and with streptozotocin and streptozotocin + polyherbal formulation $(D+P)$ for diabetic groups.

A. Graph represents step-through latency in the retention trial (STL-r) of passive avoidance learning (PAL) task in the control and diabetic groups. Columns represent Mean \pm SEM of step-through latency time (s).

B. Graph represents time spend in the dark compartment (TDC) of passive avoidance learning (PAL) task in the control and diabetic groups. Columns represent mean \pm SEM.

Tukey's multiple comparison tests was done to compare values between groups. ${ }^{* *}$ indicates $\mathrm{P}<0.001,{ }^{* *}$ indicates $\mathrm{P}<0.01,{ }^{*}$ indicates $\mathrm{P}<0.05$ and $\mathrm{ns}$ indicates $P>0.05$. \# indicates comparison of all groups with control group $C$.
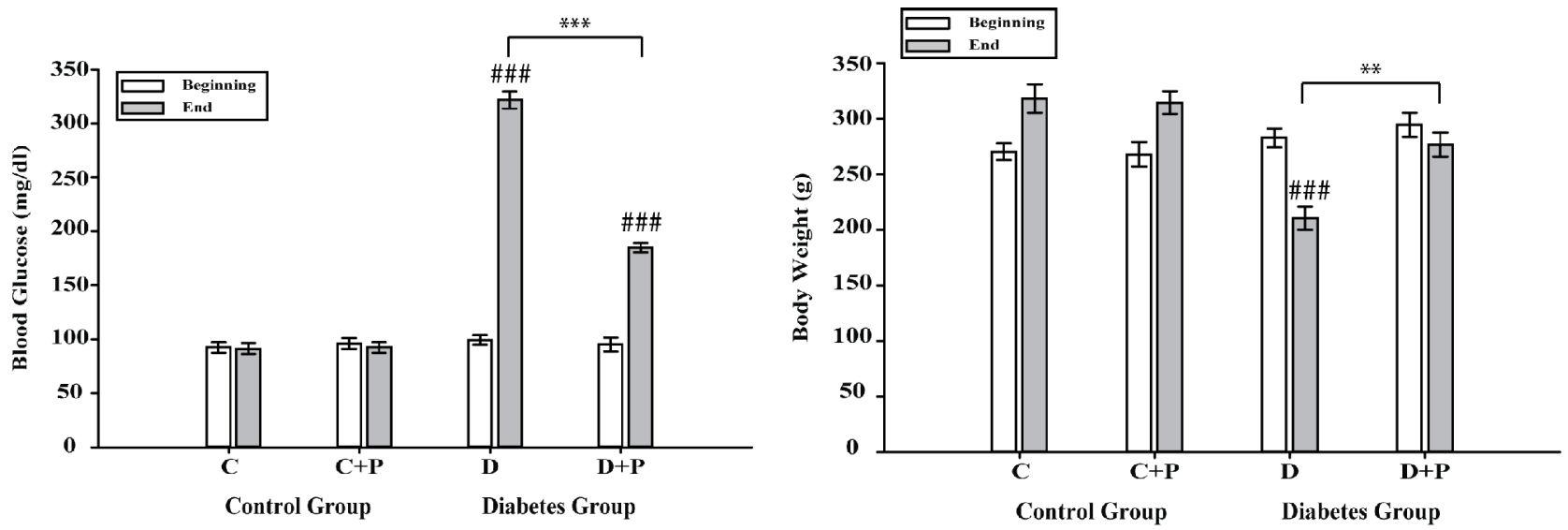

Figure 3: Effect of oral administration of polyherbal formulation on plasma glucose levels and body weight.

Blood glucose and body weight of rats were recorded at the beginning and end of the 30 day treatment period; experimental groups involved saline treated (group C) or polyherbal formulation treated (group $\mathrm{C}+\mathrm{P}$ ) control groups; streptozotocin at a dose $60 \mathrm{mg} / \mathrm{kg}$ of was used to induce diabetes which were then treated with either saline (group $\mathrm{D}$ ) or polyherbal formulation (group $\mathrm{D}+\mathrm{P}$ ) for diabetic groups.

A. Graph represents blood glucose levels at the beginning and end of the study. Columns represent Mean \pm SEM of blood glucose (mg/dl)

B. Graph represents body weight at the beginning and end of the study. Columns represent Mean \pm SEM of body weight (g).

Tukey's multiple comparison tests was done to compare values between groups. ${ }^{* * *}$ indicates $\mathrm{P}<0.001,{ }^{* *}$ indicates $\mathrm{P}<0.01,{ }^{*}$ indicates $\mathrm{P}<0.05$ and ns indicates

$P>0.05$. \# indicates comparison of all groups with control group $C$.

associated learning and memory impairments in rats to test the therapeutic potential of a novel PF. Streptozotocin at a low dose induces insulin resistant like brain state and is used widely as a method of inducing sporadic AD [37]. It produces both behavioural and neurochemical features that resembled human AD. Therefore, we evaluated the effect of PF on learning and memory deficits upon PAL paradigm in STZ diabetic rats. By monitoring the blood glucose levels, we found that STZ treatment induced diabetes in rats. In the PAL paradigm these rats showed disrupted learning and memory functions. There was a significant increase in the number of trials to acquisition, reduction in STL-r time, and increase in time spend in the dark compartment (TDC) as compared to the non-diabetic rats. However, treatment with PF containing hydro-alcoholic extracts of
Bacopa monnieri, Hippophae rhamnoides and Dioscorea bulbifera for 30 days significantly improved PAL and memory in diabetic rats. We also observed a significantly lowered blood glucose level and increased body weight of diabetic rats at the end of the treatment. In PAL acquisition task a significant decrease in the number of trials in the PF treatment groups in control and diabetic rats was evidence of improvement in learning and memory functions. Similarly in the PAL retention task, a significant increase in latency time (STLr) and decrease in TDC were indicative of memory enhancing and nootropic effects of the PF treatment.

The patented PF studied here was inspired from Indian system of traditional medicine which indicated a long history of the traditional 
use of the three plants Bacopa monnieri, Hippophae rhamnoides and Dioscorea bulbifera in the PF $[20,22,36]$. Bacopa monnieri, commonly known as bhramhi is a widely used and experimentally studied plant for improving brain functions and treatment of epilepsy and depression [51]. Studies have shown that Bacoside $\mathrm{A}$ is the main bioactive component and consists of a mixture of saponins (Bacoside A3, bacopaside II, jujubogenin isomer of bacopasaponin $\mathrm{C}$ and bacopasaponin $\mathrm{C}$ ) [52]. Several experimental studies have demonstrated the neuro-protective properties of Bacopa monnieri such as regulation of cholinergic system, enhancing acetylcholine levels, reducing neuroinflammation, amyloid levels and oxidative stress [52-57]. Hippophae rhamnoides, commonly known as sea buckthorn or amlavetas, also has been traditionally used for its medicinal properties [58]. The fruits and seeds are rich in antioxidants, carotenoids, flavonoids, organic acids, amino acids, vitamins, micro and macronutrients, phytosterols, and fatty acids omega- 3 and 6 [59-61]. Several studies have reported a wide range of medicinal properties of Hippophae rhamnoides fruit and seed preparations that include immuno-modulatory, anti-hypertensive, cardioprotective, antiglycemic, antilipidemic, anticholesterolemic, antioxidant and antiapoptotic properties [41,62-65]. Quercetin is the bioactive constituent in Hippophae rhamnoides that modulate neurotransmitter levels by inhibiting AChE and monoamine oxidase B activity [66,67]. It acts as a neuroprotective agent by reducing oxidative stress and promotes learning, memory and neuron survival [68-70]. Dioscorea bulbifera, commonly known as air potato or varahikand is widely used for its antidiabetic properties [71-73]. Extracts from this plant are rich in phenolic and flavonoid content and the major phytoconstituents are diosgenin and diosgenin acetate [74]. Studies have shown that extracts of Dioscorea bulbifera inhibit amylase and glucosidase and mediate its anti-hyperglycemic effects [73]. Other medicinal properties reported include cardio-protective, anti-oxidant, and anti-apoptotic properties [75-77]. In silico analysis have shown that diosgenin has an affinity to bind to amyloid peptides in monomeric and fibril form and can induce calcium influx in human cortical neuronal cell line [78,79]. These studies indicate a possible mechanism by which this molecule could affect neuronal structure and function.

The notable medicinal properties of the PF attest the effects we observed on learning and memory in diabetic and control rats after the treatment. This result was in accordance with our previous clinical study, in which we showed significant improvement in cognitive deficits associated with $\mathrm{AD}$ in elderly subjects [35]. The novelty of this study lies in the fact that the PF is a combination treatment, with multitargeted drug action [34]. This is in contrast with other experimental studies which focus on single targeted therapeutic action of natural compounds [25-29]. Secondly, this study supports the reverse pharmacology approach which claims to reduce costs, time and toxicity issues in drug development [80]. However there is scope to understand the mechanism of action and optimize safety, efficacy and acceptability of the PF as a drug. Further experiments are needed to study the effectiveness of the PF on different animal behavioural models such as Morris water maze test, elevated maze test, novel object recognition test etc. along with biochemical investigations to assess the antioxidant and anti-inflammatory marker levels considering the medicinal properties of the plants in PF. Additionally, the effect of PF on cholinergic deficits, taupathy, amyloid plaques should be studied to decipher the molecular basis of the therapeutic action. Since the PF is poly-molecular in nature, hence deciphering the molecular mechanisms underlying its cognitive benefits is a challenge. Future studies should utilize systems biology, in silico and high throughput genomics approaches to decipher molecular pathways affected by the PF.
Estimates indicate millions of people suffering from dementia and diabetes will increase several fold in the next millennium. These numbers suggests that as the population ages, T2DM associated cognitive dysfunction and dementia will become a huge burden on the caretakers and society. Research in the last 2 decades have provided valuable insights into understanding the pathophysiology of these diseases, however the ultimate goal is to offer patients preventive treatment that can protect them against accelerated cognitive decline. Current treatments option available for dementia are very limited (cholinesterase inhibitors, NMDA receptor antagonist) [14]; they only provide short term symptomatic relief and do not prevent disease progression. Also, over the last decade a large number of drug candidates have failed in clinical trials [15]. The paucity of treatment is due to the multi-factorial nature of diabetes associated cognitive dysfunction. There is a need to develop combination therapies, however adverse effects associated with modern synthetic drugs could prove to be prohibitive when trying to develop them as combination treatments [16,17]. The key advantage of the PF used in this study is its muti-targeted therapeutic action and lower side effects. However, clinical trials are warranted to assess its efficacy and safety in T2DM patients with cognitive dysfunction.

In conclusion, our study demonstrates that administration of a novel PF containing hydro-alcoholic extracts of Bacopa monnieri, Hippophae rhamnoides and Dioscorea bulbifera, to diabetic rats for 30 days protects against the diabetes induced cognitive deficits and reduces blood glucose levels. The PF contains multiple active constituents and may impart its therapeutic action by enhancing acetylcholine concentration, attenuating hyperglycemia, reducing oxidative stress and neuroinflammation. This treatment may be developed into a therapeutic that will provide a new potential for treatment and prevention of diabetes associated neurodegeneration and cognitive dysfunction.

\section{Acknowledgment}

This study was supported in part by Ministry of AYUSH and DST India. Additional support was provided by SRM University, Chennai and Arvind Remedies Ltd. No competing financial interests exist for any authors. All authors declare no conflicts of interest.

\section{References}

1. Ott A, Stolk RP, van Harskamp F, Pols HA, Hofman A, et al. (1999) Diabetes mellitus and the risk of dementia: The Rotterdam Study. Neurology 53: 19371942.

2. Butterfield DA, Di Domenico F, Barone E (2014) Elevated risk of type 2 diabetes for development of Alzheimer disease: A key role for oxidative stress in brain. Biochim Biophys Acta 1842: 1693-1706.

3. de la Monte SM (2014) Type 3 diabetes is sporadic Alzheimer ${ }^{3}$ s disease: minireview. Eur Neuropsychopharmacol 24: 1954-1960.

4. De La Monte SM (2012) Metabolic derangements mediate cognitive impairment and Alzheimer's disease: Role of peripheral insulin-resistance diseases. Panminerva Med. 54: 171-178.

5. de la Monte SM, Tong M (2014) Brain metabolic dysfunction at the core of Alzheimer's disease. Biochem Pharmacol 88: 548-559.

6. Gispen WH, Biessels GJ (2000) Cognition and synaptic plasticity in diabetes mellitus. Trends Neurosci 23: 542-549.

7. Rivera EJ, Goldin A, Fulmer N, Tavares R, Wands JR, et al. (2005) Insulin and insulin-like growth factor expression and function deteriorate with progression of Alzheimer's disease: link to brain reductions in acetylcholine. J Alzheimers Dis 8: $247-268$.

8. Steen E, Terry BM, Rivera EJ, Cannon JL, Neely TR, et al. (2005) Impaired insulin and insulin-like growth factor expression and signaling mechanisms in Alzheimer's disease--is this type 3 diabetes? J Alzheimers Dis 7: 63-80. 
9. Talbot K, Wang HY, Kazi H, Han LY, Bakshi KP, et al. (2012) Demonstrated brain insulin resistance in Alzheimer's disease patients is associated with IGF-1 resistance, IRS-1 dysregulation, and cognitive decline. J Clin Invest 122: 13161338.

10. Butterfield DA (2006) Oxidative stress in neurodegenerative disorders. Antioxid Redox Signal 8: 1971-1973.

11. de la Monte SM (2012) Contributions of brain insulin resistance and deficiency in amyloid-related neurodegeneration in Alzheimer's disease. Drugs 72: 49-66.

12. de la Monte SM (2012) Triangulated mal-signaling in Alzheimer's disease: roles of neurotoxic ceramides, ER stress, and insulin resistance reviewed. J Alzheimers Dis 30 Suppl 2: S231-249.

13. De la Monte SMAN, Chu J, Lawton M (2008) Epidemiological trends strongly suggest exposures as etiologic agents in the pathogenesis of sporadic Alzheimer's disease, diabetes mellitus, and non-alcoholic steatohepatitis. J Alzheimer's Dis.17: 519-529.

14. Ghezzi L, Scarpini E, Galimberti D (2013) Disease-modifying drugs in Alzheimer's disease. Drug Des Devel Ther 7: 1471-1478.

15. Cummings JL, Morstorf T, Zhong K (2014) Alzheimer's disease drugdevelopment pipeline: Few candidates, frequent failures. Alzheimers Res Ther 6: 37.

16. Giacomini KM, Krauss RM, Roden DM, Eichelbaum M, Hayden MR, et al. (2007) When good drugs go bad. Nature 446: 975-977.

17. Birks J, Harvey RJ (2006) Donepezil for dementia due to Alzheimer's disease (Cochrane Review). The Cochrane Library, Issue 3. Cochrane Libr.

18. Bennerman R, Burton J, Chen W (1983) Traditional medicine and health care coverage. Geneva, Switzerland.

19. Report of the Task Force on Conservation and Sustainable use of Medicinal Plants. Task Force on conservation and sustainable use of medicinal plants New Delhi.

20. Rao RV, Descamps O, John V, Bredesen DE (2012) Ayurvedic medicinal plants for Alzheimer's disease: A review. Alzheimers Res Ther 4: 22.

21. Dastmalchi K, Dorman HJD, Vuorela H, Hiltunen R (2007) Plants as Potential Sources for Drug Development against Alzheimer 's Disease. Int J Biomed Pharm Sci.1: 83-104.

22. Howes MJ, Houghton PJ (2003) Plants used in Chinese and Indian traditional medicine for improvement of memory and cognitive function. Pharmacol Biochem Behav 75: 513-527.

23. Perry E, Howes MJ (2011) Medicinal plants and dementia therapy: Herbal hopes for brain aging? CNS Neurosci Ther 17: 683-698.

24. Kumar GP, Khanum F (2012) Neuroprotective potential of phytochemicals. Pharmacogn Rev 6: 81-90.

25. Mao XY, Cao DF, Li X, Yin JY, Wang ZB, et al. (2014) Huperzine A ameliorates cognitive deficits in streptozotocin-induced diabetic rats. Int J Mol Sci 15: 76677683.

26. Gao C, Liu Y, Jiang Y, Ding J, Li L (2014) Geniposide ameliorates learning memory deficits, reduces tau phosphorylation and decreases apoptosis via GSK3ß Pathway in streptozotocin-induced alzheimer rat model. Brain Pathol 24: $261-269$.

27. Jabbarpour Z, Shahidi S, Saidijam M, Sarihi A, Hassanzadeh T, et al. (2014) Effect of tempol on the passive avoidance and novel object recognition task in diabetic rats. Brain Res Bull 101: 51-56.

28. Liu J, Feng L, Ma D, Zhang M, Gu J, et al. (2013) Neuroprotective effect of paeonol on cognition deficits of diabetic encephalopathy in streptozotocininduced diabetic rat. Neurosci Lett. Elsevier Ireland Ltd.549: 63-68.

29. Khan MB, Khan MM, Khan A, Ahmed ME, Ishrat T, et al. (2012) Naringenin ameliorates Alzheimer's disease (AD)-type neurodegeneration with cognitive impairment (AD-TNDCI) caused by the intra-cerebroventricular-streptozotocin in rat model. Neurochem Int 61: 1081-1093.

30. Hasanein P, Shahidi S (2010) Effects of combined treatment with vitamins C and $E$ on passive avoidance learning and memory in diabetic rats. Neurobiol Learn Mem 93: 472-478.

31. Zhuo JM, Wang H, Pratic D (2011) Is hyperhomocysteinemia an Alzheimer's disease $(A D)$ risk factor, an AD marker, or neither? Trends Pharmacol Sci 32: 562-571.
32. Galimberti D, Scarpini E (2011) Inflammation and oxidative damage in Alzheimer's disease: friend or foe? Front Biosci (Schol Ed) 3: 252-266.

33. Bossy-Wetzel E, Schwarzenbacher R, Lipton SA (2004) Molecular pathways to neurodegeneration. Nat Med 10 Suppl: S2-9.

34. Medina-Franco JL, Giulianotti MA, Welmaker GS, Houghten RA (2013) Shifting from the single to the multitarget paradigm in drug discovery. Drug Discov Today 18: 495-501.

35. Sadhu A, Upadhyay P, Agrawal A, llango K, Karmakar D, et al. (2014) Management of cognitive determinants in senile dementia of Alzheimer's type: therapeutic potential of a novel polyherbal drug product. Clin Drug Investig 34: 857-869.

36. Dubey GP (2007) Herbal preparation for management of cardiovascular and neurologic disorders, US 7273626 B2. United States: United States Patent and Trademark Office US.

37. Salkovic PM, Knezovic A, Hoyer S, Riederer P (2013) What have we learned from the streptozotocin-induced animal model of sporadic Alzheimer's disease about the therapeutic strategies in Alzheimer's research. J Neural Transm 120: 233-252.

38. Kamal A, Biessels GJ, Duis SE, Gispen WH (2000) Learning and hippocampa synaptic plasticity in streptozotocin-diabetic rats: interaction of diabetes and ageing. Diabetologia 43: 500-506.

39. Aguiar S, Borowski T (2013) Neuropharmacological review of the nootropic herb Bacopa monnieri. Rejuvenation Res 16: 313-326.

40. Tharaheswari M, Jayachandra Reddy N, Kumar R, Varshney KC, Kannan M et al. (2014) Trigonelline and diosgenin attenuate ER stress, oxidative stressmediated damage in pancreas and enhance adipose tissue PPAR? activity in type 2 diabetic rats. Mol Cell Biochem 396:161-174.

41. Suryakumar G, Gupta A (2011) Medicinal and therapeutic potential of Sea buckthorn (Hippophae rhamnoides L.). J Ethnopharmacol 138: 268-278.

42. Arison RN, Ciaccio El, Glitzer MS, Cassaro JA, Pruss MP (1967) Light and electron microscopy of lesions in rats rendered diabetic with streptozotocin. Diabetes 16: 51-56.

43. Haider S, Ahmed S, Tabassum S, Memon Z, Ikram M, et al. (2013) Streptozotocin-induced insulin deficiency leads to development of behavioral deficits in rats. Acta Neurol Belg 113: 35-41.

44. Hohenegger M, Rudas B (1971) Kidney function in experimental diabetic ketosis. Diabetologia 7: 334-338.

45. Ogren SO (1985) Evidence for a role of brain serotonergic neurotransmission in avoidance learning. Acta Physiol Scand Suppl 544: 1-71.

46. Akter K, Lanza E, Martin SA, Myronyuk N, Rua M (2011) Diabetes mellitus and Alzheimer's disease: shared pathology and treatment. Br J Clin Pharmacol.71: 365-376.

47. Francis PT, Palmer AM, Snape M, Wilcock GK (1999) The cholinergic hypothesis of Alzheimer's disease: a review of progress. J Neurol Neurosurg Psychiatry 66: 137-147.

48. de la Monte SM, Longato L, Tong M, Wands JR (2009) Insulin resistance and neurodegeneration: roles of obesity, type 2 diabetes mellitus and non-alcoholic steatohepatitis. Curr Opin Investig Drugs 10: 1049-1060.

49. Umegaki H (2014) Type 2 diabetes as a risk factor for cognitive impairment current insights. Clin Interv Aging 9: 1011-1019.

50. Frlich L, Blum-Degen D, Bernstein HG, Engelsberger S, Humrich J, et al (1998) Brain insulin and insulin receptors in aging and sporadic Alzheimer's disease. J Neural Transm 105: 423-438.

51. Dhanasekaran M, Tharakan B, Holcomb LA, Hitt AR, Young KA, et al. (2007) Neuroprotective mechanisms of ayurvedic antidementia botanical Bacopa monniera. Phytother Res 21: 965-969.

52. Deepak M, Sangli GK, Arun PC, Amit A (2005) Quantitative determination of the major saponin mixture bacoside A in Bacopa monnieri by HPLC. Phytochem Anal 16: 24-29.

53. Priyanka HP, Bala P, Ankisettipalle S, ThyagaRajan S (2013) Bacopa monnier and L-deprenyl differentially enhance the activities of antioxidant enzymes and the expression of tyrosine hydroxylase and nerve growth factor via ERK $1 / 2$ and NF-?B pathways in the spleen of female wistar rats. Neurochem Res 38:141-152. 
Citation: Upadhyay P, Sadhu A, Purohit S, Singh PK, Shivakumar S, et al. (2015) Effect of a Novel Polyherbal Formulation on Diabetes Induced Memory Deficits in Rats. Clin Exp Pharmacol 5: 194. doi:10.4172/2161-1459.1000194

54. Thomas RB, Joy S, Ajayan MS, Paulose CS (2013) Neuroprotective potential of Bacopa monnieri and Bacoside $A$ against dopamine receptor dysfunction in the cerebral cortex of neonatal hypoglycaemic rats. Cell Mol Neurobiol 33:1065-1074

55. Le XT, Pham HTN, Do PT, Fujiwara H, Tanaka K, et al. (2013) Bacopa monnieri ameliorates memory deficits in olfactory bulbectomized mice: Possible involvement of glutamatergic and cholinergic systems. Neurochem Res. 38: 2201-2015.

56. Limpeanchob N, Jaipan S, Rattanakaruna S, Phrompittayarat W, Ingkaninan K (2008) Neuroprotective effect of Bacopa monnieri on beta-amyloid-induced cell death in primary cortical culture. J Ethnopharmacol 120: 112-117.

57. Rastogi M, Ojha RP, Devi BP, Aggarwal A, Agrawal A, et al. (2012) Amelioration of age associated neuroinflammation on long term bacosides treatment. Neurochem Res 37: 869-874.

58. Patel CA, Divakar K, Santani D, Solanki HK, Thakkar JH (2012) Remedial Prospective of Hippophae rhamnoides Linn. (Sea Buckthorn). ISRN Pharmacol 2012: 436857.

59. Gumustekin K, Taysi S, Alp HH, Aktas O, Oztasan N, et al. (2010) Vitamin E and Hippophea rhamnoides $L$. extract reduce nicotine-induced oxidative stress in rat heart. Cell Biochem Funct 28: 329-333.

60. Geetha S, Ram MS, Sharma SK, Ilavazhagan G, Banerjee PK, et al. (2009) Cytoprotective and antioxidant activity of seabuckthorn (Hippophae rhamnoides $L$.) flavones against tert-butyl hydroperoxide-induced cytotoxicity in lymphocytes. J Med Food 12: 151-158.

61. Cheng JY, Teng D, Li W (2011) [Protection and mechanism of total flavone of Hippophae rhamnoides on vascular endothelial cells]. Zhongguo Zhong Xi Y Jie He Za Zhi 31: 355-358.

62. Ramasamy T, Varshneya C, Katoch VC (2010) Immunoprotective Effect of Seabuckthorn (Hippophae rhamnoides) and Glucomannan on T-2 ToxinInduced Immunodepression in Poultry. Vet Med Int 2010: 149373.

63. Pang X, Zhao J, Zhang W, Zhuang X, Wang J, et al. (2008) Antihypertensive effect of total flavones extracted from seed residues of Hippophae rhamnoides L. in sucrose-fed rats. J Ethnopharmacol. 117:325-331.

64. Wang J, Zhang W, Zhu D, Zhu X, Pang X, et al. (2011) Hypolipidaemic and hypoglycaemic effects of total flavonoids from seed residues of Hippophae rhamnoides L. in mice fed a high-fat diet. J Sci Food Agric 91: 1446-1451.

65. Basu M, Prasad R, Jayamurthy P, Pal K, Arumughan C, et al. (2007) Antiatherogenic effects of seabuckthorn (Hippophaea rhamnoides) seed oil. Phytomedicine 14: 770-777.

66. Saaby L, Rasmussen HB, Jger AK (2009) MAO-A inhibitory activity of quercetin from Calluna vulgaris (L.) Hull. J Ethnopharmacol 121: 178-181.
67. Chimenti F, Cottiglia F, Bonsignore L, Casu L, Casu M, et al. (2006) Quercetin as the active principle of Hypericum hircinum exerts a selective inhibitory activity against MAO-A: Extraction, biological analysis, and computational study. J Nat Prod.69:945-949.

68. Ishisaka A, Ichikawa S, Sakakibara H, Piskula MK, Nakamura T, et al. (2011) Accumulation of orally administered quercetin in brain tissue and its antioxidative effects in rats. Free Radic Biol Med 51: 1329-1336.

69. Ansari MA, Abdul HM, Joshi G, Opii WO, Butterfield DA (2009) Protective effect of quercetin in primary neurons against Abeta(1-42): relevance to Alzheimer's disease. J Nutr Biochem 20: 269-275.

70. Zerin T, Kim YS, Hong SY, Song HY (2013) Quercetin reduces oxidative damage induced by paraquat via modulating expression of antioxidant genes in A549 cells. J Appl Toxicol 33: 1460-1467.

71. Rgo Tde S, Ash Lda S, Pessoa L, Feij MB, Leite J, et al. (2014) The intake of yam (Dioscorea bulbifera Linn) attenuated the hyperglycemia and the bone fragility in female diabetic rats. Nutr Hosp 29: 370-375.

72. Singh RG, Rajak M, Ghosh B; Usha, Agrawal A, Dubey GP (2013) Comparative evaluation of fosinopril and herbal drug Dioscorea bulbifera in patients of diabetic nephropathy. Saudi J Kidney Dis Transpl 24: 737-742.

73. Ghosh S, Ahire M, Patil S, Jabgunde A, Dusane MB, (2012) Antidiabetic activity of Gnidia glauca and Dioscorea bulbifera: Potent amylase and glucosidase inhibitors. Evidence-based Complement Altern Med:10.

74. Ghosh S, Derle A1, Ahire M1, More P1, Jagtap S1, et al. (2013) Phytochemical analysis and free radical scavenging activity of medicinal plants Gnidia glauca and Dioscorea bulbifera. PLoS One 8: e82529.

75. Chen X, Wu SH, Zeng XB, Jiang XW, Chen XP, et al. (2013) Antioxidant and SGC-7901 cell inhibition activities of Rhizoma Dioscoreae bulbiferae. ethanol extracts. Afr J Tradit Complement Altern Med 10: 261-266.

76. Tohda C, Urano T, Umezaki M, Nemere I, Kuboyama T (2012) Diosgenin is an exogenous activator of 1,25D,f-MARRS/Pdia3/ERp57 and improves Alzheimer's disease pathologies in 5XFAD mice. Sci Rep 2: 535.

77. Jayachandran KS, Vasanthi HR, Rajamanickama GV (2010) Flavonoid rich fraction of Dioscorea bulbifera Linn. (Yam) enhances mitochondrial enzymes and antioxidant status and thereby protects heart from isoproterenol induced myocardial infarction. Curr Pharm Biotechnol.11:887-894.

78. Ngo ST, Li MS (2013) Top-leads from natural products for treatment of Alzheimer's disease: docking and molecular dynamics study. Mol Simul 39:279-291.

79. Wang YJ, Liu YC, Chang HD, Wu SN (2006) Diosgenin, a plant-derived sapogenin, stimulates $\mathrm{Ca} 2+$-activated $\mathrm{K}^{+}$current in human cortical $\mathrm{HCN}-1 \mathrm{~A}$ neuronal cells. Planta Med 72:430-436.

80. Patwardhan B, Vaidya ADB, Mukund C, Joshi SP (2008) Reverse pharmacology and systems approaches for drug discovery and development. Curr Bioact Compd. 4:1573-4072. 